\title{
Clinical Spectrum and Medical Comorbidities in Tuberculosis: A Hospital-Based Study in Northeast India
}

Prasanta Bhattacharya ${ }^{1}$, Kishore Talukdar ${ }^{1}$, Bhupen Barman ${ }^{1}$, Md Jamil ${ }^{1}$, Pranjal Phukan ${ }^{2}$, Habung Mobing ${ }^{1}$, Gwenette War ${ }^{1}$, Phibakordor L. Nonglait ${ }^{1}$, Subrahmanya Murti ${ }^{3}$, Konthoujam Prithviraj Sr. ${ }^{1}$, Bandi Sangma ${ }^{4}$

1. Department of General Medicine, North Eastern Indira Gandhi Regional Institute of Health and Medical Sciences (NEIGRIHMS), Shillong, IND 2. Department of Radiology, North Eastern Indira Gandhi Regional Institute of Health and Medical Sciences (NEIGRIHMS), Shillong, IND 3. Department of Cardiology, Smt. Nathiba Hargovandas Lakhmichand (NHL) Municipal Medical College (NHLMMC), Ahmedabad, IND 4. Department of Chest \& Tuberculosis, North Eastern Indira Gandhi Regional Institute of Health and Medical Sciences (NEIGRIHMS), Shillong, IND

Corresponding author: Bhupen Barman,drbhupenb@gmail.com

\section{Abstract}

\section{Background}

Tuberculosis (TB) is one of the most common infectious diseases and is commonly associated with comorbidities. However, data regarding TB and comorbidities are lacking from northeast India. The aim of the study is to see the clinical spectrum of TB and the frequency of comorbidities.

\section{Methods}

This was a prospective observational study of all hospitalized TB patients between January 2016 and June 2017 who were selected by consecutive sampling. Data were analyzed using SPSS v. 17.0 (IBM Corp., Armonk, $\mathrm{NY}$ ), and a p-value of $<0.05$ was considered significant.

\section{Results}

Of the 173 patients selected, the mean age was $41.05 \pm 17.04$ years with a male:female ratio of 4.27:1. Pulmonary TB (PTB) was found in $43.94 \%$, extra-pulmonary TB (EPTB) in $52.02 \%$, and disseminated TB in $4.04 \%$. Fever $(61.27 \%)$ was the most common presentation, followed by cough $(54.33 \%)$ and breathlessness (32.94\%). Of the 76 patients with PTB and seven with disseminated TB, making a total of 83 patients, 56 (67.4\%) were sputum positive. Out of 90 patients suffering from EPTB, pleural effusion (53.33\%) was the commonest type of EPTB, followed by central nervous system (CNS) tuberculosis (26.66\%) and abdominal tuberculosis (8.88\%). Comorbidities were present in $53.17 \%$ of the patients, of which diabetes mellitus (DM) (26.58\%) and hypertension (17.34\%) were the most common. Comorbid conditions were significantly higher in PTB than EPTB ( 51 of 83 vs. 41 of 90, p<0.05). Mean glycated hemoglobin (HbA1c) was significantly higher in PTB as compared to ЕРТВ (8.74 \pm 2.04 vs. $7.58 \pm 0.29$, p<0.05).

Review began 09/10/2020 Review ended 09/16/2020 Published 09/21/2020

\section{() Copyright 2020}

Bhattacharya et al. This is an open access article distributed under the terms of the Creative Commons Attribution License CC-BY 4.0., which permits unrestricted use, distribution, and reproduction in any medium, provided the original author and source are credited.

\section{Conclusion}

Comorbidities, particularly DM, were present in half of the patients, mostly in PTB than EPTB, with glycemic control being significantly poorer in PTB patients.

Categories: Endocrinology/Diabetes/Metabolism, Internal Medicine, Infectious Disease

Keywords: pulmonary tuberculosis (ptb), extrapulmonary tuberculosis (eptb), diabetes mellitus (dm), anti tubercular therapy (att)

\section{Introduction}

India accounts for one-fourth of the global TB burden of tuberculosis (TB) in the world, with an estimated 2.8 million cases in 2015 [1]. About $40 \%$ of the Indian population is infected with TB, the majority having a latent infection, which can potentially flare up into active disease. While extra-pulmonary tuberculosis (EPTB) accounts for a quarter of the annual incidence of TB globally, the overall literature available regarding the spectrum of EPTB is limited.

Most countries with a high TB burden also face a concomitant burden of associated medical comorbidities. These include liver diseases, chronic kidney diseases (CKD), diabetes mellitus (DM), cardiovascular diseases, connective tissue disorders, chronic obstructive pulmonary disease, and so on. Such medical comorbidities interact with TB at multiple levels. On one hand, these may aggravate the tubercular process from latent to active TB or even to disseminated forms, may cause diagnostic challenges, and lead to ineffective treatment. On the other hand, these comorbidities may restrict the use of some potent anti-tubercular drugs. 
Furthermore, TB itself can aggravate the comorbidities or hinder in the diagnosis and/or optimum management of such comorbidities.

Published data on TB and associated comorbidities from the North-Eastern states of India are limited. Out of a total of 14,24,771 newly notified cases of TB in India in 2015, 53,427 cases were from the northeastern states, with Assam accounting for the majority with 36724 cases followed by Meghalaya (3,934 cases) and the least from Sikkim (1,463 cases) [1]. With this background, this study was planned to study the clinical spectrum of TB and the association of comorbidities in patients attending a tertiary care center in northeast India, which caters to most of the patients in the state.

\section{Materials And Methods}

This hospital-based prospective observational study was conducted for all cases of tuberculosis admitted to the department of general medicine between January 1, 2016, and December 31, 2016. During the study period, 654 tuberculosis patients got registered at the DOTS (directly observed treatment, short-course) center for antitubercular therapy (ATT). A total of 173 patients admitted during the study period in the department of general medicine were included in the study group by the consecutive sampling method. Ethical clearance was taken from the Institutional Ethics Committee prior to the commencement of the study. The diagnosis of TB was made in all the cases fulfilling the following criteria:

- Sputum positive TB

- Sputum negative TB but conventional chest radiography suggestive of TB

- Fluid cytology suggestive of TB

• Fine needle aspiration cytology (FNAC)/biopsy confirmation of TB

- Computed tomography (CT) scan (contrast or non-contrast)/magnetic resonance imaging (MRI) scan suggestive of TB

Information was collected after obtaining valid informed consent. The socio-demographical, clinical, and laboratory parameters and outcomes were assessed. The demographic data included were age, sex, ethnic and occupational background. Clinical data comprised symptoms related to the primary etiology of tuberculosis and its predisposing factors and/or associated comorbidities. Laboratory investigations included complete hemogram, sputum analysis for acid-fast bacilli (AFB), blood sugar estimation, blood urea, serum creatinine, serum electrolytes, liver function tests, coagulation profile, and routine urine analysis. Wherever necessary, fluid cytology, FNAC, biopsy, urine for AFB, chest X-ray, ultrasonography (USG) of the abdomen, and other ancillary biochemical and radiological investigations were done.

\section{Statistical analysis}

To describe the characteristics of the study population, Microsoft Excel 2010 (Microsoft Corporation, Redmond, Washington) was used. SPSS Statistics version 17.0 (IBM Corp., Armonk, NY) was used to perform the statistical analysis. Continuous data were presented as mean (with SD) and the categorical data were expressed as count (with percentage). Analyses of categorical variables were performed by the chi-square test. A p-value of $<0.05$ was considered significant.

\section{Results}

The case group consisted of 173 patients (age $=41.05 \pm 17.04$ years) of which the majority were males $(80.93 \%)$, with a male to female ratio of $4.27: 1$. The most common age group was $18-30$ years for both males and females. Out of the 173, 76 (43.94\%) patients were pulmonary tuberculosis (PTB), 90 (52.02\%) patients were extra-pulmonary tuberculosis (EPTB), and the remaining seven (4.04\%) were disseminated tuberculosis. All cases of disseminated tuberculosis in the present study had pulmonary involvement. For the purposes of comparison between PTB and ЕPTB, disseminated cases were considered in the PTB bracket. Among seven cases of disseminated tuberculosis, five had comorbid HIV infection, with all patients having a cluster of differentiation 4 (CD4) count of less than 50 cells/ $\mu \mathrm{L}$. Both PTB and EPTB were more common in males as compared to females. Further, among 138 male patients, PTB ( 71 of 138 , i.e. 51.44\% was more common than ЕPTB, but among 35 female patients, EPTB (28 of 35 i.e. 80\%) was more common than PTB. The mean age in PTB patients ( $42.86 \pm 16.2$ years) was higher than in EPTB patients ( $38.94 \pm 17.45$ years); the difference being statistically insignificant. The average duration of hospital stay in patients with TB was $7.59 \pm 4.72$ days. The mean duration of hospitalization in РТВ, ЕРТВ, and disseminated TB were $6.2 \pm 4.75$ days, $6.96 \pm 4.8$ days, and $13.28 \pm 5.12$ days, respectively.

The most common symptomatic presentation among our patients was low-grade fever, with an evening rise of temperature (61.27\%) followed by cough (54.33\%), breathlessness (32.94\%), and altered sensorium (17.64\%) (Table 1). The most common predisposing behavioral risks observed in this study were smoking (59.53\%), alcohol abuse (56.64\%), and tobacco (42.19\%). Alcohol use was significantly higher in PTB as 


\section{Cureus}

compared to EPTB $(\mathrm{p}<0.05)$. Nineteen point sixty-five percent $(19.65 \%)$ of the patients did not have any such predisposing factor (Table 2). Out of 90 patients suffering from EPTB, tubercular pleural effusion (53.33\%) was the commonest type of EPTB, followed by CNS tuberculosis (26.66\%) and abdominal tuberculosis (8.88\%) (Table 3).

\begin{tabular}{|c|c|c|c|c|}
\hline \multirow{2}{*}{ Presenting symptom } & \multicolumn{4}{|c|}{ Number of patients $(\%)$} \\
\hline & PTB $(n=76)$ & EPTB $(n=90)$ & Disseminated $=7$ & Total $(n=173)$ \\
\hline Abdominal distension & 1 & 8 & - & $9(5.20)$ \\
\hline Altered sensorium & 1 & 22 & 4 & $27(17.64)$ \\
\hline Breathlessness & 20 & 35 & 2 & $57(32.94)$ \\
\hline Chest discomfort & 10 & 13 & - & $23(13.29)$ \\
\hline Chest pain & 7 & 8 & - & $15(8.67)$ \\
\hline Chronic diarrhea & 0 & 2 & 1 & $3(1.73)$ \\
\hline Cough & 63 & 31 & - & $94(54.33)$ \\
\hline Fever & 57 & 49 & - & $106(61.27)$ \\
\hline Generalized weakness & 10 & 7 & - & $17(9.82)$ \\
\hline Hemoptysis & 17 & 0 & - & $17(9.82)$ \\
\hline Jaundice & 1 & 11 & - & $12(6.93)$ \\
\hline Oliguria & 1 & 5 & - & $6(3.46)$ \\
\hline Weight loss & 3 & - & 3 & $8(4.62)$ \\
\hline
\end{tabular}

TABLE 1: Showing presenting symptoms of patients with tuberculosis

\begin{tabular}{|l|l|l|l|l|l|}
\hline \hline Behavioral risk & PTB $(\mathbf{n}=\mathbf{7 6})$ & EPTB $(\mathbf{n = 9 0})$ & Disseminated $=(\mathbf{n}=\mathbf{7})$ & Total $(\mathbf{n}=\mathbf{1 7 3})$ & P-value (PTB+disseminated vs EPTB) \\
\hline Smoking & 50 & 51 & 2 & $103(59.53)$ & $\mathrm{p}>0.05$ \\
Alcohol consumption & 53 & 40 & 5 & $98(56.64)$ & $\mathrm{P}<0.05$ \\
Tobacco chewing & 30 & 38 & 5 & $73(42.19)$ & $\mathrm{P}>0.05$ \\
IV drug users & 1 & 1 & 2 & $4(2.31)$ & $\mathrm{P}>0.05$ \\
High risk sexual history & 2 & 0 & 2 & $4(2.31)$ & $\mathrm{P}<0.05$ \\
None & 15 & 18 & 1 & $34(19.65)$ & $\mathrm{P}>0.05$ \\
\hline
\end{tabular}




\section{Cureus}

\begin{tabular}{|l|lll|}
\hline Type of EPTB & \multicolumn{2}{l|}{ Number of patient (\%) } & \\
\hline Abdominal tuberculosis & Male & Female & Total \\
\hline CNS tuberculosis & 5 & 3 & $8(8.88)$ \\
\hline Pericardial effusion & 16 & 8 & $24(26.66)$ \\
Pleural effusion & 1 & 2 & $3(3.33)$ \\
\hline Spine tuberculosis & 33 & 15 & $48(53.33)$ \\
\hline Tubercular lymphadenopathy & 1 & 1 & $2(2.73)$ \\
\hline Total & 3 & 2 & $5(5.07)$ \\
\hline
\end{tabular}

TABLE 3: Showing the incidence of different types of EPTB

CNS: central nervous system; EPTB: extra-pulmonary tuberculosis

In the present study, various medical comorbidities were present in $53.17 \%$ of the patients, of which diabetes mellitus (26.58\%) and hypertension (17.34\%) were the most common. Comorbid conditions were significantly higher in PTB than EPTB ( 51 of 83 vs. 41 of 90, p<0.05). Also, the mean age of patients with comorbidity was significantly higher than those without comorbidities $(46.12 \pm 18.02$ vs. $34.42 \pm 16.51$, $p<0.05)$. All the comorbid conditions, except for ischemic heart disease, were more common in PTB as compared to EPTB, however, diabetes mellitus and chronic kidney disease were significantly higher in PTB as compared to EPTB (Table 4). Mean HbA1c was significantly higher in PTB as compared to EPTB (8.74 2.04 vs. $7.58 \pm 0.29, \mathrm{p}<0.05)$. 


\section{Cureus}

\begin{tabular}{|c|c|c|c|c|c|}
\hline Comorbidity & PTB + disseminated $n=83$ & EPTB $\mathbf{N}=90$ & Total $(\%$ of $n=173)$ & Mean age (years) & P-value \\
\hline Diabetes mellitus & 35 & 11 & 46 (26.58) & 45.96 & $\mathrm{P}<0.05$ \\
\hline Hypertension & 16 & 14 & 27 (17.34) & 54 & $p>0.05$ \\
\hline Ischemic heart disease & 1 & 3 & $4(2.31)$ & 48.5 & $p>0.05$ \\
\hline Chronic liver disease & 9 & 8 & $17(9.82)$ & 44.33 & $p>0.05$ \\
\hline Chronic kidney disease & 5 & 1 & $6(3.46)$ & 44.5 & $P<0.05$ \\
\hline Chronic obstructive pulmonary disease & 3 & 3 & $6(3.46)$ & 57.67 & $p>0.05$ \\
\hline Connective tissue disorder & 2 & 0 & $2(1.15)$ & 25 & $p>0.05$ \\
\hline Hypothyroidism & 4 & 3 & $6(3.46)$ & 43.5 & $p>0.05$ \\
\hline \multirow{2}{*}{$\mathrm{HIV}^{*}$} & 5 & 0 & $5(2.89)$ & 29.4 & - \\
\hline & Overall PTB + disseminated & Overall EPTB & & & \\
\hline Comorbidity & 51 & 41 & $92(53.17)$ & $46.12 \pm 18.02$ & \\
\hline No comorbidity & 32 & 49 & $81(46.83)$ & $34.42 \pm 16.51$ & \\
\hline P-value & & & 0.03 & 0.04 & \\
\hline Grand total & 83 & 90 & 00) & & \\
\hline
\end{tabular}

\section{TABLE 4: Showing distribution of comorbid medical conditions}

*all HIV comorbid patients are disseminated tuberculosis

PTB: pulmonary tuberculosis; EPTB: extra-pulmonary tuberculosis; HIV: human immunodeficiency virus

Of the 76 (43.94 \%) patients suffering from pulmonary tuberculosis and seven (4.04\%) with disseminated tuberculosis, making a total of 83 patients, 56 (67.4\%) were sputum positive, the rest being sputum negative. Of the seven patients with disseminated tuberculosis, only two (28.57\%) were sputum positive - one male and female, respectively. On comparing sputum-positive patients (56) to sputum-negative patients (27), pulmonary tuberculosis, comorbid conditions ( 36 of 56 vs. 18 of 27 , p>0.05), presence of diabetes ( 20 of 56 vs. 15 of $27, p>0.05)$, and mean $\operatorname{HbA1c}(8.95 \pm 1.6$ vs. $8.16 \pm 3.41$, p>0.05) were higher in sputum-positive PTB, but the results were not statistically significant (Table 5). 


\section{Cureus}

\begin{tabular}{|c|c|c|c|c|}
\hline \multirow{2}{*}{ Type of PTB } & \multicolumn{3}{|c|}{ Number of patients (\%) } & \multirow{2}{*}{ P-value } \\
\hline & Comorbidity & No comorbidity & Total & \\
\hline Sputum positive (SP) & 36 & 20 & 56 & \multirow{2}{*}{0.07} \\
\hline Sputum negative (SN) & 18 & 9 & 27 & \\
\hline Total & 54 & 29 & 83 & - \\
\hline \multirow{2}{*}{ Type of PTB } & \multicolumn{3}{|c|}{ Number of patients (\%) } & \multirow{2}{*}{ P-value } \\
\hline & Diabetes & No diabetes & Total & \\
\hline SP & 20 & 36 & 56 & \multirow{2}{*}{0.08} \\
\hline SN & 15 & 12 & 27 & \\
\hline Total & 35 & 48 & 83 & - \\
\hline
\end{tabular}

\section{TABLE 5: Showing comparisons between sputum-positive and sputum-negative PTB}

ATT was given to all patients, of which 135 (78.03\%) were given Category I ATT (61 (45.18\%) had PTB and 74 (54.82\%) had EPTB) and the remaining 38 patients were given Category II ATT (22 (57.89\%) had PTB and 16 (42.01\%) had EPTB). On a comparison between retreatment cases (i.e. those on Category II) as compared to new cases, the proportion of PTB was significantly higher in the re-treatment group $(\mathrm{P}<0.05)$.

\section{Discussion}

In the present study, the majority of patients were male (80.93\%) with a male to female ratio of $4.27: 1$. The mean age was $41.05 \pm 17.04$ years with the most common age group being $18-30$ years. Worldwide, including the latest Indian data, males have a higher prevalence of tuberculosis [1]. The exact reason for the same is still a subject of active research [2]. The most common age group for tuberculosis in India is the third decade as per the latest Revised National TB Control Program (RNTCP) data. This is also reflected in a large systematic review, as well as a large population-based study from Europe [3-4]. However, there is a rising trend of tuberculosis in the elderly, as reflected in national and international data [5]. In the present study, EPTB was higher in females than males as against the norm of male preponderance. The same trend of female preponderance in EPTB, particularly pleural effusion, was also shown in a recent gap analysis of south Asian studies on EPTB [6].

Among predisposing behavioral risks, smoking (59.53\%) was most commonly found in patients with tuberculosis followed by alcohol consumption (56.64\%) and tobacco chewing (42.19\%). Smoking has long been linked to tuberculosis. The first strong relation was proposed in a systematic review in 2005, with evidence becoming stronger from a recent population-based study from China that showed a two-fold risk of pulmonary tuberculosis with smoking [7-8]. Alcohol use has also been associated with tuberculosis with a large systematic review suggesting a causal association [9]. A recent meta-analysis has shown that alcohol use led to 2.35 deaths and 22.02 incident cases per 1,00,000 people from tuberculosis in 2014 [10].

In the present study, 27 out of 83 (32.53\%) pulmonary tuberculosis patients were sputum negative. Sputumnegative pulmonary tuberculosis is a diagnostic and treatment challenge wherein patients have radiologically active lesions with no evidence of acid-fast bacilli in the sputum. These patients may be further culture positive or negative. Sputum-negative pulmonary tuberculosis has been linked to a high incidence of HIV coinfection in a population-based study from Brazil [11]. Further, in a population-based study from the Netherlands, patients with smear-negative, culture-positive tuberculosis were responsible for $13 \%$ of TB transmission [12]. This highlights the importance of this subset of TB patients. In the present study, a majority (68.63\%) of all extrapulmonary cases were tubercular pleural effusions followed by abdominal tuberculosis. This is in conformity with most Indian data as from Karnataka and Himachal Pradesh [13-14]. However, a study from Bhopal found a higher incidence of TB lymphadenitis as compared to a pleural effusion [15].

In the present study, various medical comorbidities were present in $53.17 \%$ of the patients, of which diabetes mellitus (26.58\%) and hypertension (17.34\%) were the most common. Comorbid conditions had a 
significantly higher association with PTB than EPTB. Diabetes mellitus (DM) and chronic kidney disease (CKD) had a significantly higher association with PTB than EPTB. Mean HbA1c was also significantly higher in PTB as compared to EPTB. Diabetes and tuberculosis have been well-recognized as intersecting epidemics. The presence of diabetes has been shown as an independent risk factor for death in TB in a large retrospective cohort study [16]. Tuberculosis and chronic kidney disease are now recognized as syndetic [17]. Tuberculosis has shown both to be a risk factor for chronic kidney disease, as well as being more prevalent in the community with chronic kidney disease from an Indian study [18-19].

Chronic liver disease (CLD) and cardiovascular disease (CVD) were also more common in PTB than EPTB, with the difference being insignificant. Liver cirrhosis has also shown to be a risk factor for TB. In a large nationwide cohort study from Taiwan, 957 of 41,076 cirrhotic patients developed TB, which was significantly higher than the 955 of 204,244 noncirrhotic patients $(\mathrm{P}<0.001)$ [20]. Tuberculosis has been associated with an increased CVD risk. Another nationwide cohort study with a three-year follow-up from Taiwan has shown that the presence of TB confers a higher risk of ischemic stroke [21]. Also, another study has shown that latent TB infection is independently associated with a higher risk of acute myocardial infarction [22].

\section{Limitation}

As the study was done in a tertiary care center, where the types of patients were mostly in the moderate to severe category with multiple comorbidities, there are chances of an inherent bias being present.

\section{Conclusions}

Tuberculosis was more common in young males. Among PTB patients, sputum-positive cases were twice as common as sputum-negative cases while pleural effusion was the most common type of EPTB. Medical comorbidities were found in nearly half the patients, being significantly more common among PTB than EPTB. Diabetes mellitus was the most common co-morbidity, which was more common and associated with significantly poorer glycaemic control in PTB as compared to EPTB. A significantly higher proportion of retreatment cases had PTB.

\section{Additional Information \\ Disclosures}

Human subjects: Consent was obtained by all participants in this study. Institution Ethics Committee, North Eastern Indira Gandhi Regional Institute of Health and Medical Sciences issued approval NEIGR/IEC/2015/0040. Informed consent has been taken from each participant. Protocol Title: Clinical spectrum and medical co-morbidities in tuberculosis - a hospital-based study in northeast India, has been recommended from IEC Committee. Animal subjects: All authors have confirmed that this study did not involve animal subjects or tissue. Conflicts of interest: In compliance with the ICMJE uniform disclosure form, all authors declare the following: Payment/services info: All authors have declared that no financial support was received from any organization for the submitted work. Financial relationships: All authors have declared that they have no financial relationships at present or within the previous three years with any organizations that might have an interest in the submitted work. Other relationships: All authors have declared that there are no other relationships or activities that could appear to have influenced the submitted work.

\section{References}

1. TB India 2017. RNTCP annual status report . (2017). Accessed: September 3, 2020: https://tbcindia.gov.in/WriteReadData/TB\%20India\%202017.pdf.

2. Zhao Y, Ying H, Demei J, Xie J: Tuberculosis and sexual inequality: the role of sex hormones in immunity . Crit Rev Eukaryot Gene Expr. 2012, 22:233-241. 10.1615/critreveukargeneexpr.v22.13.60

3. Donald P, Marais B, Barry CE 3rd: Age and the epidemiology and pathogenesis of tuberculosis . Lancet. 2010, 375:1852-1854. 10.1016/S0140-6736(10)60580-6

4. Zhang X, Andersen AB, Lillebaek T, et al.: Effect of sex, age, and race on the clinical presentation of tuberculosis: a 15-year population-based study. Am J Trop Med Hyg. 2011, 85:285-290. 10.4269/ajtmh.2011.10-0630

5. Negin J, Abimbola S, Marais BJ: Tuberculosis among older adults - time to take notice. Int J Infect Dis. 2015, 32:135-137. 10.1016/.ijid.2014.11.018

6. Mehraj J, Khan ZY, Saeed DK, Shakoor S, Hasan R: Extrapulmonary tuberculosis among females in South Asia-gap analysis. Int J Mycobacteriol. 2016, 5:392-399. 10.1016/j.ijmyco.2016.09.054

7. Bothamley GH: Smoking and tuberculosis: a chance or causal association? . Thorax. 2005, 60:527-528. 10.1136/thx.2004.036012

8. Yen YF, Yen MY, Lin YS, et al.: Smoking increases risk of recurrence after successful anti-tuberculosis treatment: a population-based study. Int J Tuberc Lung Dis. 2014, 18:492-498. 10.5588/ijtld.13.0694

9. Rehm J, Samokhvalov AV, Neuman MG, et al.: The association between alcohol use, alcohol use disorders and tuberculosis (TB). A systematic review. BMC Public Health. 2009, 9:450. 10.1186/1471-2458-9-450

10. Imtiaz S, Shield KD, Roerecke M, Samokhvalov AV, Lönnroth K, Rehm K: Alcohol consumption as a risk factor for tuberculosis: meta-analyses and burden of disease. Eur Respir J. 2017, 50:1700216. 10.1183/13993003.00216-2017

11. Campos LC, Rocha MV, Willers DM, et al.: Characteristics of patients with smear-negative pulmonary 
tuberculosis (TB) in a region with high TB and HIV prevalence. PLoS One. 2016, 11:0147933.

10.1371/journal.pone.0147933

12. Tostmann A, Kik SV, Kalisvaart NA, Sebek MM, Verver S, Boeree MJ, van Soolingen D: Tuberculosis transmission by patients with smear-negative pulmonary tuberculosis in a large cohort in the Netherlands. Clin Infect Dis. 2008, 47:1135-1142. 10.1086/591974

13. Prakasha SR, Suresh G, D'sa IP, Shetty SS, Kumar SG: Mapping the pattern and trends of extrapulmonary tuberculosis. J Glob Infect Dis. 2013, 5:54-59. 10.4103/0974-777X.112277

14. Gupta A: Clinico-epidemiological profile of extra pulmonary tuberculosis: a report from a high prevalence state of northern India. Int J Epidemiol. 2015, 44:127-128. 10.1093/ije/dyv096.119

15. Shrivastava AK, Brahmachari S, Pathak P, Kumar R, Sainia T, Patel U, Mandil A: Clinico-epidemiological profile of extra-pulmonary tuberculosis in central India. Int J Med Res Rev. 2015, 3:223-230. 10.17511/ijmrr.2015.i2.046

16. Degner NR, Wang JY, Golub JE, et al.: The effect of diabetes and comorbidities on tuberculosis treatment outcomes. Am J Respir Crit Care Med. 2017, 195:7587.

17. Romanowski K, Clark EG, Levin A, Cook VJ, Johnston JC: Tuberculosis and chronic kidney disease: an emerging global syndemic. Kidney Int. 2016, 90:34-40. 10.1016/j.kint.2016.01.034

18. Shen TC, Huang KY, Chao CH, et al.: The risk of chronic kidney disease in tuberculosis: a population-based cohort study. QJM. 2015, 108:397-403. 10.1093/qjmed/hcu220

19. Venkata RK, Kumar S, Krishna RP, Kumar SB, Padmanabhan S, Kumar S: Tuberculosis in chronic kidney disease. Clin Nephrol. 2007, 67:217-220. 10.5414/cnp67217

20. Lin YT, Wu PH, Lin CY, et al.: Cirrhosis as a risk factor for tuberculosis infection--a nationwide longitudinal study in Taiwan. Am J Epidemiol. 2014, 180:103-110. 10.1093/aje/kwu095

21. Sheu JJ, Chiou HY, Kang JH, Chen Y-H, Lin H-C: Tuberculosis and the risk of ischemic stroke. A 3-year follow-up study. Stroke. 2010, 41:244-249. 10.1161/STROKEAHA.109.567735

22. Huaman MA, Ticona E, Miranda G, et al.: The relationship between latent tuberculosis infection and acute myocardial infarction. Clin Infect Dis. 2018, 66:886-892. 10.1093/cid/cix910 